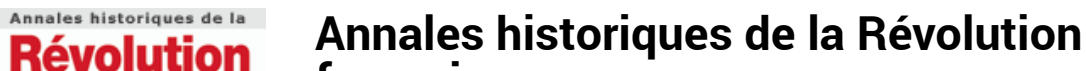 \\ française française
}

324 | avril-juin 2001

Louis Charles Antoine Desaix. Officier du roi, Général de la République

\section{Desaix devant Thèbes}

\section{Sadek Néaimi}

\section{(2) OpenEdition \\ Journals}

Édition électronique

URL : https://journals.openedition.org/ahrf/371

DOI : 10.4000/ahrf.371

ISSN : 1952-403X

Éditeur :

Armand Colin, Société des études robespierristes

Édition imprimée

Date de publication : 1 juin 2001

Pagination : 63-67

ISSN : 0003-4436

\section{Référence électronique}

Sadek Néaimi, «Desaix devant Thèbes », Annales historiques de la Révolution française [En ligne], 324 | avril-juin 2001, mis en ligne le 22 mai 2006, consulté le 24 avril 2022. URL : http:// journals.openedition.org/ahrf/371 ; DOI : https://doi.org/10.4000/ahrf.371

Ce document a été généré automatiquement le 24 avril 2022.

Tous droits réservés 


\title{
Desaix devant Thèbes
}

\author{
Sadek Néaimi
}

1 Quelle est la perspective égyptienne sur l'expédition française en Égypte (1798 - 1801)? Elle condamne tout à fait l'acte militaire et colonial et voit quelquefois, sans fondement, dans cette campagne une continuité avec les croisades, posant que Bonaparte est un autre Saint Louis, sans rendre compte de la rupture radicale de la France de la fin du xviiie siècle avec la pensée médiévale, une rupture due en grande partie aux philosophes du siècle des Lumières. Comment cependant concilier le génie de la liberté que la France hérite de Montesquieu, de Voltaire, de Diderot, eux-mêmes hostiles, à différents égards, à la colonisation, et cette action coloniale et militaire ? Et cette question en entraîne une seconde: comment se fait-il que la Révolution française s'achève ainsi sur un projet colonial? Que cette France qui au milieu du XVIIIe siècle exportait des philosophes envoie à la fin du même siècle des militaires occuper la terre des autres?

2 Dialogue de sourds. Du côté égyptien on en reste à la simple condamnation; du côté français on insiste sur l'aspect positif de l'expédition, justifiant d'une façon ou d'une autre le projet colonial comme une action destinée à libérer de leurs despotes les peuples opprimés, à diffuser la civilisation et le génie de la liberté. D'un côté on condamne, sans réfléchir aux causes qui dans le passé ont permis la colonisation, très voisines de celles qui permettent aujourd'hui la néocolonisation. De l'autre on produit une histoire destinée à satisfaire les sentiments nationaux. De la sorte, on ne favorise pas l'esprit de dialogue et d'ouverture à l'autre, et l'on n'arrive jamais à tenir un discours équilibré : comme dans ces émissions de télévision qui, pour attirer l'attention de spectateurs paresseux, recherchent le merveilleux, l'insolite, et quelquefois chez l'autre le mauvais côté des choses. Une attitude que l'on trouve déjà dans le regard des voyageurs des XVIe, XVIIe et XVIIIe siècles cherchant à nourrir leur imagination en décrivant l'insolite chez autrui.

3 S'en tenir à cette superficialité ne nous permettrait pas de comprendre l'activité déployée par Desaix en Égypte, avec une assiduité remarquable. Durant les neuf mois passés en tant que chef de l'armée française en haute Égypte il a parcouru avec sa division, en état de guerre, souvent à pied, cinq mille kilomètres, avec des chaussures et 
des habits déchirés. Sans épiloguer cependant sur le côté militaire de l'aventure, insistons davantage sur les aspects intellectuels et humains de sa personnalité manifestés pendant les vingt mois qui vont du 2 juillet 1798 au 3 mars 1800 . Son humanité nous est pour une part révélée par ces deux mamelouks (appellation polie en arabe pour dire esclaves), l'un circassien, l'autre africain noir, qui l'accompagnèrent de son arrivée au Caire à sa mort à Marengo, le 14 juin 1800. Au soir de la bataille, devant le cadavre du général dont le cœur avait été déchiré par une balle, les deux hommes, Ismaël et Bakel, ont pleuré, avec une affliction témoigant de la générosité des sentiments que Desaix leur avait inspirés. En revanche, Bonaparte qui devait pourtant beaucoup à Desaix, devant son corps resta avare de ses larmes. Pourquoi deux mamelouks, égyptianisés par leur intégration dans la société égyptienne, ont-ils versé spontanément les leurs à la vue de Desaix mort? Ne fournissent-ils pas une bonne occasion de chercher chez l'autre les «bons usages » et l'équité de conduite qui a pu être ressentie par les Égyptiens de haute Égypte au temps de ce Desaix qu'ils appelèrent «le sultan juste »?

4 L'un de ces aspects positifs est que l'Égypte doit à Desaix, dans une certaine mesure, la renaissance du mouvement moderne de traduction - dans les deux sens - entre l'arabe et le français. Dès son arrivée au Caire le 23 juillet 1798, deux jours après la bataille des Pyramides, Desaix prend contact avec Dom Raphaël, un nom entendu au Vatican après que Bonaparte l'ait chargé, avec le mathématicien Monge - futur membre de la Commission des Arts et des Sciences du Caire - de mettre la main sur le matériel de l'imprimerie arabe utilisée par l'Église romaine pour son prosélytisme. La mission incluait aussi le recrutement d'interprètes parmi les étudiants en langues orientales : dans ce groupe se distinguaient deux Syriens de rite grec-catholique qui ont pu connaître et signaler ensuite à l'attention des enquêteurs, leur coreligionnaire Dom Raphaël, comme on l'appelle dans l'historiographie égyptienne. Cet Égyptien catholique d'origine syrienne maîtrisait le français et l'italien. Avec l'expédition son nom jusque-là obscur prend de l'importance : le voici promu membre de l'Institut d'Égypte, section "Arts et Littérature » créée par un arrêté signé Bonaparte le 22 août 1798. Ce choix s'explique par sa bonne connaissance du français et de sa langue maternelle, non par sa confession catholique.

5 La tendance déiste et laïque des Français qui dirigent l'expédition - si l'on excepte Kléber, assassiné au Caire par un Syrien le jour même de la mort de Desaix - a favorisé la coopération des chrétiens orthodoxes égyptiens, autrement dit des coptes. Cette même tendance a également réussi à détourner Dom Raphaël d'une vie consacrée à la religion. Dès lors, on assiste au début d'un mouvement de traduction de textes autres que religieux, comme le mémoire de Desgenettes, le médecin en chef de l'expédition, intitulé Avis sur la petite vérole régnante, publié en version bilingue, arabe et française. Malgré sa tendance hostile aux idées des Lumières qui apparaît dans quelques notices sans grande importance intitulées Notices sur la mort de Voltaire, et d'autre part sa conviction qu'il n'y a pas de point commun entre les Français de cette expédition et l'esprit des croisades, Dom Raphaël accepte ainsi, au nom du pouvoir colonisateur, de s'allier à des dirigeants français eux-mêmes emplis des idées de Voltaire et des encyclopédistes. Peut-être parce que le pouvoir a plus de force d'attraction que la religion.

6 Lorsque la nouvelle de la mort de Desaix parvient en Égypte, le 14 septembre 1800, en geste de fidélité à sa mémoire et selon un conseil du Moalem Jacob, Dom Raphaël 
compose en trois jours un poème de condoléances : quarante vers en arabe qu'il traduit lui-même en français. Sans doute ce poème n'a-t-il pas grande valeur poétique : à l'image de ce qu'est le plus souvent la poésie arabe de l'époque, du fait de la décadence de la langue sous la domination turque. Mais sa traduction française est envoyée au général Abdella Menou - Jacques Menou jusqu'à sa conversion à l'Islam à la suite de son mariage avec une belle Égyptienne de Rosette - troisième commandant en chef de l'armée française en Égypte, à l'intention du gouvernement français, à Paris. On peut voir dans ce geste une preuve de l'humanité reconnue à Desaix dans ses missions civiles. Du point de vue militaire on a retenu l'indulgence du général envers un garçon de douze ans, dans une ville que l'historiographie égyptienne dénomme El-Fokay, Belliard et Vivant Denon El-Fachen. On amène à Desaix un petit garçon en criant: «C'est un voleur, il a volé des fusils aux volontaires, on l'a pris!» Et apparaît un bel enfant, blessé au bras d'un large coup de sabre, qui regarde sa blessure sans émotion. Il se présente d'un air naïf et confiant au général qu'il reconnaît pour son juge. On lui demande qui lui a dit de voler ces fusils : «Personne ». S'il a des parents : «Une mère seulement, bien pauvre et aveugle ». Assis sous un arbre, le général lui dit que s'il avoue qui l'a envoyé on ne lui fera rien; que s'il s'obstine à se taire, il sera puni comme il le mérite. «Je vous l'ai dit, personne ne m'a envoyé. Dieu seul m'a inspiré ». Puis, jetant son bonnet aux pieds de Desaix : "Voilà ma tête, faites-la couper ». Le général dit: «Pauvre petit malheureux! Qu'on le renvoie ». Le jeune garçon vit que son arrêt était prononcé, il regarda Desaix et partit avec le sourire de la confiance, un sourire dû à l'indulgence de Desaix.

7 Cette anecdote est symbolique de la façon dont on interprète l'histoire. En France elle est portée au crédit de Desaix, tandis que les historiens égyptiens y voient la preuve d'une résistance à laquelle prenaient part même les enfants, avec un courage qui leur permettait d'affronter seuls et sans armes l'ennemi de leur pays. Mais du coup ils sont conduits à négliger la bienveillance pacifique du général étranger envers un enfant, attitude qui reconnaît implicitement la légitimité de la volonté de résistance du jeune Égyptien.

8 Passons à un autre aspect bénéfique de la présence de Desaix. La campagne de HauteÉgypte a un intérêt culturel important, mais indirect. Encouragé et protégé par Desaix, Vivant Denon a fait des efforts remarquables pour la découverte, l'analyse et la compréhension de la civilisation pharaonique. Des efforts à considérer comme des jalons, à la suite de travaux comme ceux de Kircher et de Warburton, dans la construction de l'égyptologie : une science qui va naître en 1822 avec Champollion, un quart de siècle après la venue de Desaix en Égypte et qui doit en partie sa naissance au crayon de Denon, aux fusils de Desaix. Sous leur protection Denon a pu décrire et dessiner des sites aujourd'hui complètement disparus : ainsi a-t-il ouvert la voie aux savants de l'expédition pour que ceux-ci réalisent leur œuvre gigantesque, la Description de l'Égypte, qui renferme une connaissance profonde et nouvelle de l'Égypte, aussi bien antique que moderne.

9 Certes, si Desaix et son armée sont allés jusqu'au fond de la haute Égypte, c'est à la poursuite de Mourad Bey, le chef des mamelouks, donc pour des raisons militaires et non pour l'amour des monuments et des œuvres d'art. C'est l'intérêt militaire qui a entraîné Desaix jusqu'à Thèbes, mission qui lui valut l'envoi par Bonaparte d'un sabre portant l'inscription « Bataille de Sediman » (bataille livrée le 7 octobre 1798 à Sedmen, dans le Faïoum). Mais l'Égypte n'était pas n'importe quel champ de bataille. Sur le 
poignard enrichi de diamants que Desaix reçut ensuite du général en chef on lisait d'un côté : « Napoléon à Desaix, vainqueur de la haute Égypte ». Et de l'autre : "Thèbes aux cent portes, Sésostris le Grand »... L'allusion au pharaon Senousert III, de la douzième dynastie du Moyen-Empire, pour partie constructeur de Thèbes, est révélatrice d'un état d'esprit. Les hasards de la guerre avaient conduit l'armée française aux deux temples de Karnak et de Louxor, dans la Vallée des rois et des reines, mais ce n'est pas par hasard que, devant le temple de Karnak et devant l'obélisque qui orne aujourd'hui à Paris la place de la Concorde, Desaix et son armée applaudissent une civilisation disparue $^{1}$. À la vue de ces ruines imposantes et de ces grands monuments, en contraste avec la décadence de l'Égypte moderne, doit-on imaginer Desaix tombant dans une réflexion profonde, à la manière de celle de Volney dans Les Ruines ? La mort précoce du général nous a privés de lire les mémoires où il aurait pu écrire ce qu'il avait alors vraiment ressenti.

\section{BIBLIOGRAPHIE}

Ghislaine Alleaume, L'expédition de Bonaparte vue d'Égypte (textes introduits par), Égypte/Monde arabe, $\mathrm{n}^{\circ} 1, \mathrm{CEDEJ}$, éditions Complexe, Bruxelles,1999.

Abd Al-Aziz Al-Shanawy, Suwar min dur el Azhar fi muquawamat al-ihtilal al-firnisi Limisr fi axakhir alqarn althamin ashar (Aspects du rôle d'al-azhar dans la résistance à l'occupation du XVIIIe siècle), Le Caire, 1971.

Jean-Marie Carré, Voyageurs et écrivains français en Égypte, 2 vol., Le Caire, IFAO, 1956.

François Charles-Roux, Les origines de l'expédition d'Égypte, Paris, 1910.

Al Jabarti Abd al-Rahman, Tarickh Muddat al-Fransis bi, Misr (Histoire des Français en Égypte), Le Caire, 1978. Partiellement reproduit dans Vivant Denon / Abdel Rahman el-Gabarti, Sur l'expédition de Bonaparte en Égypte, témoignages croisés et commentés par Mahmoud Hussein, Actes Sud, Babel, 1998.

Henri Laurens, Kléber en Égypte, Kléber et Bonaparte, Le Caire, IFAO, 1988.

L'expédition d'Égypte, Paris, Armand Colin, 1989.

Les Origines intellectuelles de l'expédition d'Égypte, l'orientalisme islamisant en France, 1698-1798, éditions Isis, Institut français d'études anatoliennes, Istanbul-Paris, 1987.

R. A. Saad, Le journal de Vivant Denon, thèse de magistère présentée par Menoufeycha (Égypte), non publiée, mais consultable, 1998.

Dominique Vivant Denon, voir ci-dessous, note 1.

\section{NOTES}

1.Dominique Vivant Denon, Voyage dans la Basse et la Haute-Égypte, Paris, Gallimard, 1998, p. 194. 


\section{RÉSUMÉS}

Vue par les autochtones, l'expédition française en Égypte aide le plus souvent à construire une histoire nationale. Celle-ci ignore les moments d'humanité chez Desaix. La clémence du «sultan juste» envers un jeune rebelle est ainsi oubliée : l'acte de l'adolescent devient emblématique d'un refus généralisé, jusqu'aux enfants, de la présence française. Rien n'est dit non plus de l'attachement des mamelouks Ismaël et Bakel au général français, pas plus que des avancées culturelles dans le domaine des traductions franco-arabes ou de l'égyptologie - il est vrai seconde par rapport aux buts militaires de la conquête.

\section{Desaix before Thebes.}

As seen by the natives, the French expedition to Egypt served to construct their national history. Instances of humanity in Desaix are ignored. Thus, the mercy of a "just sultan" towards a young rebel is forgotten : the action of the youngster becomes emblematic of the general rejection, even by the children, of the French presence. No mention is made either of the affection of the Mamelukes Ismaël and Bakel for the French general, or the cultural progress achieved in the fields of French-Arabic translation or Egyptology, admittedly secondary goals compared to the prime aim of military conquest.

\section{INDEX}

Mots-clés : campagne d'Égypte, colonie, Institut du Caire, égyptologie, historiographie

\section{AUTEUR}

\section{SADEK NÉAIMI}

Université du Caire 